\title{
Efficacy of seven and fourteen days of antibiotic treatment in uncomplicated Staphylococcus aureus bacteremia (SAB7): study protocol for a randomized controlled trial
}

Louise Thorlacius-Ussing $^{1 *}$ (D), Christian Østergaard Andersen², Niels Frimodt-Møller ${ }^{3}$, Inge Jenny Dahl Knudsen², Jens Lundgren ${ }^{4}$ and Thomas Lars Benfield ${ }^{1}$

\begin{abstract}
Background: Staphylococcus aureus bacteremia $(S A B)$ is frequently encountered in the hospital setting, and current guidelines recommend at least 14 days of antibiotic treatment for SAB in order to minimize risks of secondary deep infections and relapse. However, evidence to support these treatment recommendations remains scarce. Patients with uncomplicated $S A B$ are known to have a low of risk of recurrence and death. Reducing treatment length in uncomplicated SAB would reduce the total consumption of antibiotics, duration of hospital admission, and potentially the risk of adverse events. With SAB7 we seek to determine if 7 days of antibiotic treatment in patients with uncomplicated SAB is non-inferior to 14 days of treatment.

Methods/design: The study is designed as a randomized, non-blinded, non-inferiority, multicenter interventional study. Primary measure of outcome will be 90-day survival without clinical or microbiological failure to treatment or relapse. Secondary outcomes include the prevalence of severe adverse effects, in particular secondary infection with Clostridium difficile, all-cause mortality, as well as public health related costs. Patients identified with uncomplicated SAB who have received 7 days of protocol-approved antibiotics will be eligible for inclusion and randomized 1:1 in two parallel arms to either (i) discontinue antibiotic treatment at day 7 or (ii) to continue antibiotic treatment for a total of 14 days. Main exclusion criteria include signs of complicated SAB, such as the presence of secondary deep infections, persistent bacteremia, and implantable devices. Patients are followed for 6 months with clinical examinations, consecutive blood tests, and registration of adverse events. A total of 284 patients are to be included at ten centers across Denmark. The primary endpoint will be tested with a statistical non-inferiority margin of 10 percentage points.
\end{abstract}

Discussion: $S A B 7$ will determine if 7 days of antibiotic treatment in patients with uncomplicated $S A B$ is sufficient and safe. Results of the study will provide important knowledge on optimized SAB management and could potentially modify the current treatment recommendations.

Trial registration: ClinicalTrails.gov, H-17027414. Registered on May 2, 2018. The Danish Medicines Agency (EudraCT), 2017-003529-13. Registered on October 30, 2017.

Keywords: Randomized controlled trial, Staphylococcus aureus bacteremia, Bloodstream infection, Treatment duration, Short-course therapy, Uncomplicated Staphylococcus aureus bacteremia

\footnotetext{
* Correspondence: louise.thorlacius-ussing@regionh.dk

1 Department of Infectious Diseases, Hvidovre Hospital, Copenhagen

University Hospital, Kettegaard Alle 30, 2650 Hvidovre, Denmark

Full list of author information is available at the end of the article
}

(c) The Author(s). 2019 Open Access This article is distributed under the terms of the Creative Commons Attribution 4.0 International License (http://creativecommons.org/licenses/by/4.0/), which permits unrestricted use, distribution, and reproduction in any medium, provided you give appropriate credit to the original author(s) and the source, provide a link to the Creative Commons license, and indicate if changes were made. The Creative Commons Public Domain Dedication waiver (http://creativecommons.org/publicdomain/zero/1.0/) applies to the data made available in this article, unless otherwise stated. 


\section{Background}

\section{Background}

The extensive use of antibiotics worldwide is closely related to the increasing issues of antimicrobial resistance and antibiotic-associated infections [1,2]. Long-course antibiotic treatment, as used for blood stream infections, plays a critical role in this context.

Staphylococcus aureus is one of the most frequent causes of bacterial infections in skin, bone, tissue, and blood, with bacteremia being one of the most severe presentations. In Denmark the incidence is around 25 per 100,000 population, corresponding to approximately 1800 cases of $S$. aureus bacteremia (SAB) each year [3-5]. SAB is often associated with severe complications, such as secondary deep infections, and a reported mortality rate of $20-25 \%$ [5]. However, nearly half of cases are classified as uncomplicated infections associated with a significantly lower risk of relapse and death [6, 7]. Uncomplicated SAB is partly due to in-hospital infections related to invasive procedures and the use of peripheral and central venous catheters, as well as community-acquired skin and soft tissue infections $[8,9]$.

Present international and Danish guidelines recommend a standard treatment regimen of a minimum of 14 days of intravenous antibiotics for SAB [6]. However, these recommendations are based on only a few randomized clinical studies and, to a greater extent, on individual expert opinion [10]. Only one minor clinical trial has evaluated the length of treatment in $\mathrm{SAB}$, and this found no significant differences in outcome between 2 and 4 weeks of treatment [11]. It is known from case-based studies that 10-14 days of treatment in uncomplicated $\mathrm{SAB}$ are associated with few secondary complications and failure to treat events [12-15]. Interestingly, three observational studies showed that 7 days of treatment in simple catheter-associated SAB were effective and not associated with a higher risk of recurrence if the focus of the infection was eradicated. In all reported cases, follow-up blood cultures were negative and the patients showed no clinical or biochemical signs of adverse events or complications $[6,7,16]$. However, present evidence regarding length of treatment for $\mathrm{SAB}$ is limited and further research in the area could potentially modify current clinical practice.

\section{Hypothesis and aims}

We hypothesize that 7 days of antibiotic treatment in uncomplicated $\mathrm{SAB}$ is non-inferior to 14 days of treatment.

We primarily seek to compare 7 and 14 days of antibiotic treatment in patients with uncomplicated SAB in terms of mortality and the prevalence of microbiological and clinical failure to treat and recurrence within 90 days of diagnosis.
The study aims to explore the possibility of reducing the consumption of antibiotics, as well as shortened hospital admission, and thereby potentially decrease the risk of adverse events and microbial resistance development.

\section{Primary and secondary outcomes Primary outcome}

- The primary outcome is 90-day survival without clinical or microbiological failure to treatment or relapse in patients treated with 7 days versus 14 days of antibiotic therapy

\section{Secondary outcomes}

- All-cause mortality on days 14, 28, 90, and 180

- Microbiological failure to treatment

- Microbiological relapse

- Clinical failure to treatment

- Desirability of outcome ranking (DOOR)

- Hospital admissions during follow-up

- Severe adverse events grade $\geq 3$

- Acute renal injury

- Clostridium difficile infection

- Infection with multidrug-resistant organisms

- Health-associated costs associated the treatment lengths

\section{Methods/design \\ Design and randomization}

The study is a randomized, non-blinded, non-inferiority, interventional study. Confirmation of study eligibility will be performed by entering key variables into a web-based program (REDCap) with subsequent automatic patient randomization into two parallel arms (ratio 1:1): treatment regimens of 7 or 14 days, respectively. Randomization lists will be generated centrally in random blocs and stratified according to catheter-associated infections as well as center/hospital. For a detailed description of the items included in the SAB7 study protocol, please see the SPIRIT table in Additional file 1.

\section{Ethics and regulatory considerations}

The study has been approved by the relevant regulatory authorities, including The Danish Medicines Agency (EudraCT 2017-003529-13), Research Ethics Committee of the Capital Region of Denmark (H-17027414), and The Danish Data Protection Registry (ID AHH-2017-086, I-Suite no.: 05891). The study will be conducted according to ICH-GCP (guidelines for Good Clinical Practice (GCP)) and monitored by GCP units in Denmark. Informed consent is obtained from all study participants. 


\section{Setting}

The following ten hospitals, representing all five regions of Denmark, will be invited to participate in the study: Hvidovre Hospital, Rigshospitalet, Herlev Hospital, Hillerød Hospital, Roskilde Hospital, Odense University Hospital, Kolding Hospital, University Hospital of Aarhus, Herning Hospital, and University Hospital of Aalborg.

\section{Study population}

Uncomplicated SAB is defined according to the guidelines of the Infectious Disease Society of America [17]. Briefly, patients are required to be free of signs of infectious endocarditis and have a negative follow-up blood culture, a physical examination without signs of metastatic foci, absence of fever 48-72 h after initiation of appropriate antibiotic therapy, and no implantable devices. Prosthetics in joint and bones implanted $>6$ months prior to $\mathrm{SAB}$ will be accepted if the patients show no clinical signs of infection involving the prosthetics. Inclusion and exclusion criteria are listed in detail in Table 1. Eligible patients must fulfill all of the inclusion and none of the exclusion criteria (Fig. 1). Cases are identified through the respective departments of microbiology and infectious diseases at each center. All participants are hospitalized at enrollment.

\section{Treatment}

Patients will receive antibiotic treatment adhering to local and national guidelines as well as according to the susceptibility of the respective isolate. Participation in the study will only affect treatment duration and will have no influence on the choice of treatment with respect to type and dose of antibiotic agent. Patients entering the study have already received 7 days of antibiotic treatment. According to randomization, patients will (i) discontinue antibiotic treatment at day 7 or (ii) continue antibiotic treatment for a total of 14 days. Antibiotics considered appropriate for empiric treatment of SAB are listed in Table 2.

\section{Assessment and data collection}

A summary of collected data is presented in Fig. 3. Study participants will be followed by doctors at the respective centers for 6 months after the SAB diagnosis (Figs. 2 and 3). Hospitalized patients will be assessed in accordance with the study protocol and local hospital standards. Day 0 is defined as the initiation of antibiotic therapy with antimicrobial effects against SAB (Fig. 1). Days $1-6$ serve as a screening period to determine study eligibility. Eligible patients will be included in the trial and randomized at day 7. To evaluate outcome efficacy patients will undergo three follow-up examinations on day 14 and weeks 12 and 26, respectively. The first follow-up visit will consist of a physical examination, whereas the second and third follow-up visits will consist of a clinical consultation or a standardized telephone interview (Fig. 2). In addition to the scheduled follow-up visits, patients will at discharge be thoroughly instructed

Table 1 Inclusion and exclusion criteria

\begin{tabular}{|c|c|}
\hline Inclusion criteria & Exclusion criteria \\
\hline - Age $>18$ years & $\begin{array}{l}\text { - Persistence of S. aureus bacteremia before randomization (S. aureus positive } \\
\text { follow-up blood culture obtained within } 48-120 \text { h of the first positive blood } \\
\text { culture) }\end{array}$ \\
\hline - Blood culture positive for Staphylococcus aureus & - Polymicrobial infection \\
\hline $\begin{array}{l}\text { - Antibiotic treatment with antimicrobial activity to S. aureus } \\
\text { administrated within } 12 \mathrm{~h} \text { of the first positive blood culture }\end{array}$ & $\begin{array}{l}\text { - Antibiotic treatment with no antimicrobial activity to } \mathrm{S} \text {. aureus administered } \\
\text { more than } 12 \mathrm{~h} \text { after the first positive blood culture }\end{array}$ \\
\hline - Temperature $<37,5^{\circ} \mathrm{C}$ at randomization & $\begin{array}{l}\text { - Endocarditis or other intracardiac infection demonstrated with transthoracic } \\
\text { or transesophageal echocardiography }\end{array}$ \\
\hline $\begin{array}{l}\text { - S. aureus negative follow-up blood culture obtained 48-120 h } \\
\text { after microbiologically verified SAB }\end{array}$ & - Previous history of endocarditis \\
\hline \multirow[t]{10}{*}{ - Patients written consent obtained } & - Pacemaker or other intracardiac implant \\
\hline & $\begin{array}{l}\text { - Failure to remove a likely focus of infection, such as central venous catheter, } \\
\text { within } 72 \mathrm{~h} \text { of the first positive blood culture }\end{array}$ \\
\hline & - Vascular grafts \\
\hline & - Pneumonia or infection involving bone, joints, or prosthetics \\
\hline & - Previous bone/joint infection \\
\hline & - S. aureus infection within the last 90 days \\
\hline & - Pregnancy or breastfeeding \\
\hline & - Neutropenia (blood neutrophils < $1.0 \times 10^{9} /$ I) \\
\hline & - Untreated cancer \\
\hline & - Chemotherapy within 90 days. \\
\hline
\end{tabular}




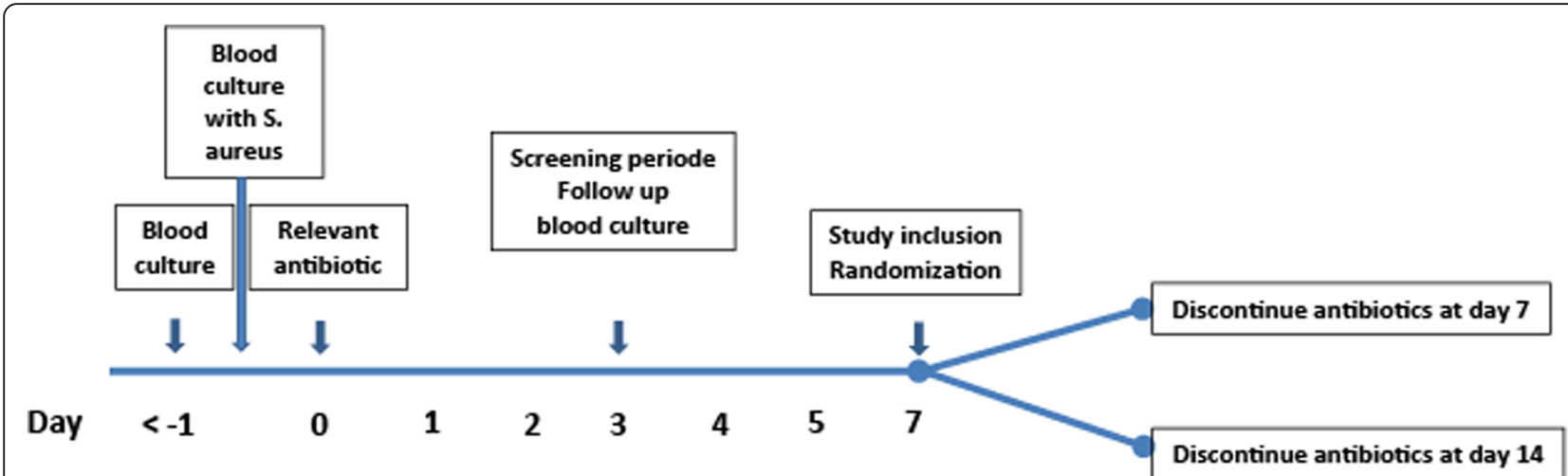

Fig. 1 Timeline of SAB7

on contacting the treating physician if they develop symptoms or fever at any time between follow-up visits. Any signs of fever must lead to a clinical evaluation, blood testing, and blood culture. Follow-up data and laboratory tests are described in Fig. 3. Additional follow-up regarding comorbidity and other clinical variables is conducted by the use of hospital and national databases. All data are registered in an electronic case report form.

\section{Outcome measures}

\section{Primary outcome}

Data regarding the composite primary endpoint, 90-day survival without clinical or microbiological failure to treatment or relapse, will be acquired through patient interviews, clinical consultation, and microbiological tests as well as hospital and national administrative registries.

Microbiological failure to treatment is defined as verified $S$. aureus, of the same genotype (based on spa type) as the initial infection, less than 7 days after treatment termination.

Microbiological SAB relapse is defined as demonstration of the same $S$. aureus genotype as the initial infection more than 7 days after treatment termination, demonstrated by molecular-based typing [18, 19]. Verification of $S$. aureus as the infectious agent in question will be performed by microbiological analysis of biological material such as blood, synovial fluid, etc.

Table 2 Acceptable empiric antibiotic treatment of $S A B$

\begin{tabular}{|c|c|c|c|c|}
\hline Antibiotic & Form * (oral or iv) & Standard dose * & Frequency * & Dose adjustment * \\
\hline Piperacillin + tazobactam (MSSA) & IV & $4 g+0.5 g$ & Every $8 \mathrm{~h}$ & Renal impairment \\
\hline Dicloxacillin (MSSA) & Oral or IV & $1 \mathrm{~g}$ & Every $6 \mathrm{~h}$ & Weight \\
\hline Flucloxacillin (MSSA) & Oral or IV & $1 \mathrm{~g}$ & Every 6-8h & Renal impairment \\
\hline Cloxacillin (MSSA) & Oral or IV & $1 \mathrm{~g}$ & Every 6-8h & Renal impairment \\
\hline Cefuroxim (MSSA) & IV & $\begin{array}{l}750 \mathrm{mg} \\
1.5 \mathrm{~g}\end{array}$ & $\begin{array}{l}\text { Every } 6 \mathrm{~h} \\
\text { Every } 8 \mathrm{~h}\end{array}$ & Renal impairment \\
\hline Clindamycin (MSSA + MRSA) & Oral & $600 \mathrm{mg}$ & Every 6-8h & - \\
\hline \multicolumn{5}{|l|}{ Macrolides (MSSA) } \\
\hline Claritromycin & Oral or IV & $500 \mathrm{mg}$ & Every $12 \mathrm{~h}$ & Renal and liver impairment \\
\hline Vancomycin (MSSA + MRSA) & IV & $1 \mathrm{~g} \mid \mathrm{V}$ & Every $12 \mathrm{~h}$ & $\begin{array}{l}\text { Renal impairment } \\
\text { Se- vanco }\end{array}$ \\
\hline Linezolid (MRSA + MSSA) & Oral or IV & $600 \mathrm{mg}$ & Every $12 \mathrm{~h}$ & - \\
\hline Rifampicin (MSSA) & Oral & $300-600$ mg & Every $8 \mathrm{~h}$ & - \\
\hline Meropenem (MSSA) & IV & $1-2 g$ & Every $8 \mathrm{~h}$ & Renal impairment \\
\hline Moxifloxacin (MSSA) & Oral or IV & $400 \mathrm{mg}$ & Every $24 \mathrm{~h}$ & - \\
\hline \multicolumn{5}{|l|}{ Aminoglycosides (MSSA) } \\
\hline -Gentamycin & IV & $5 \mathrm{mg} / \mathrm{kg}$ & Every $24 \mathrm{~h}$ & Renal impairment \\
\hline
\end{tabular}

*Standard recommendations

IV intravenous, MSSA methicillin-sensitive Staphylococcus aureus, MRSA methicillin-resistant Staphylococcus aureus, Se-vanco serum-vancomycin 


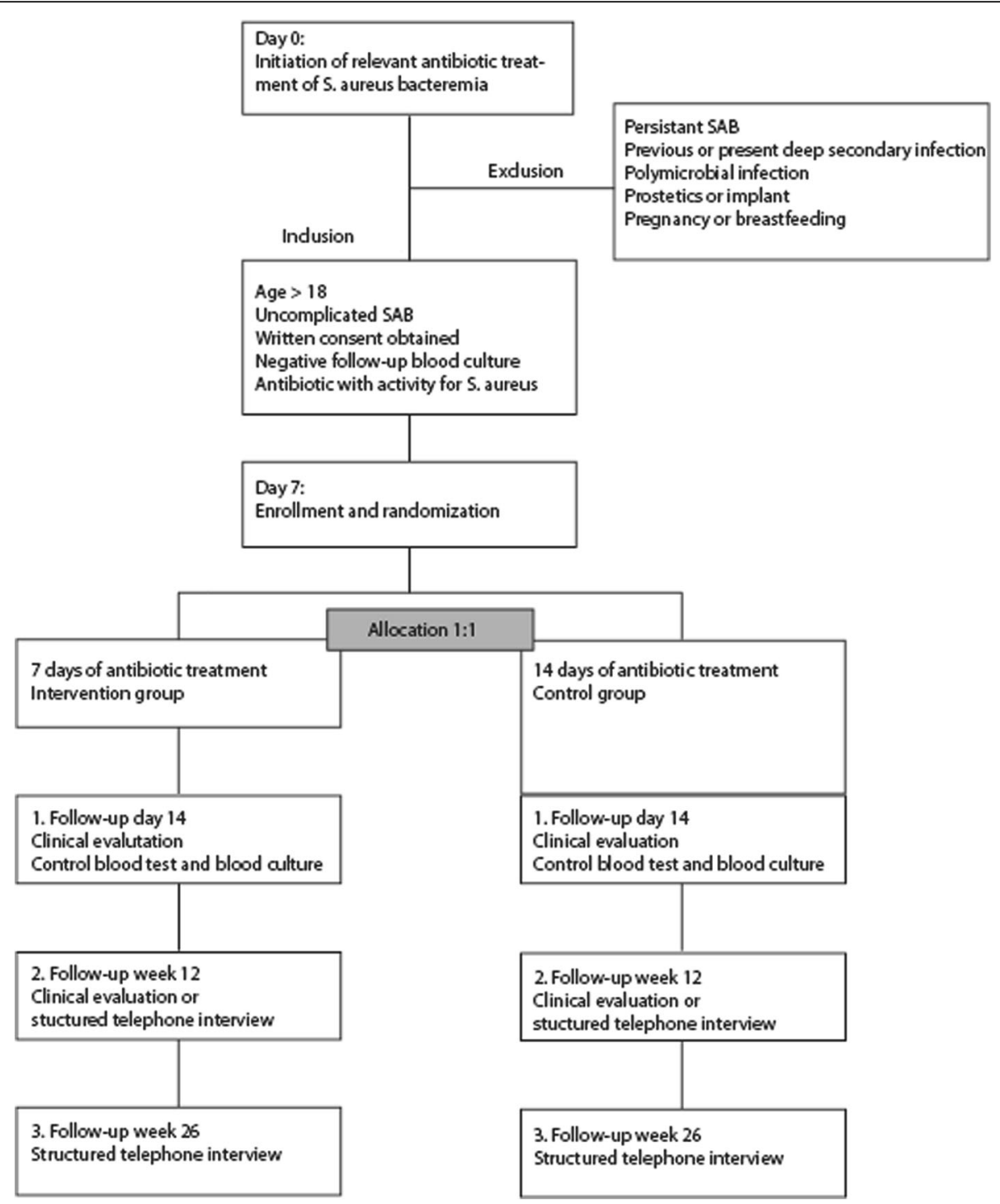

Fig. 2 Flowchart of SAB7

Clinical failure to treatment or relapse is defined as initiation of anti-staphylococcal therapy for more than $48 \mathrm{~h}$ on suspected clinical recurrence.

Data on vital status during follow-up are obtained from local hospital registries and the Danish Civil Registration System.

Data regarding the primary endpoint will be evaluated and determined by an independent committee (Endpoint Review Committee) blinded to randomization.

\section{Secondary outcome}

The components of the composite primary outcome will also be assessed separately as a secondary outcome, which is defined as described above. Additional secondary objectives are overall survival at days 14, 28 , and 180 and the presence of severe adverse events, including the prevalence of $C$. difficile-associated diarrhea, infection with a multidrug-resistant organism and grade 3 or above adverse events as defined elsewhere (https://ctep.cancer.gov/protocoldevelopment/ electronic_applications/ctc.htm). The prevalence of adverse events will be derived from patient interviews and laboratory reports, including, if relevant, documentation of the microbiological method applied for verification of C. difficile. Additionally, follow-up blood tests will also be used to assess adverse events by screening for possible antibiotic-related affects on, e.g., liver and kidney function. Acute kidney injury is defined as a 1.5-fold increase in plasma creatinine or a $25 \%$ decrease of estimated glomerular filtration rate (GFR).

Our definition of multidrug-resistant organisms will rely on identification of resistant bacteria in a clinical 


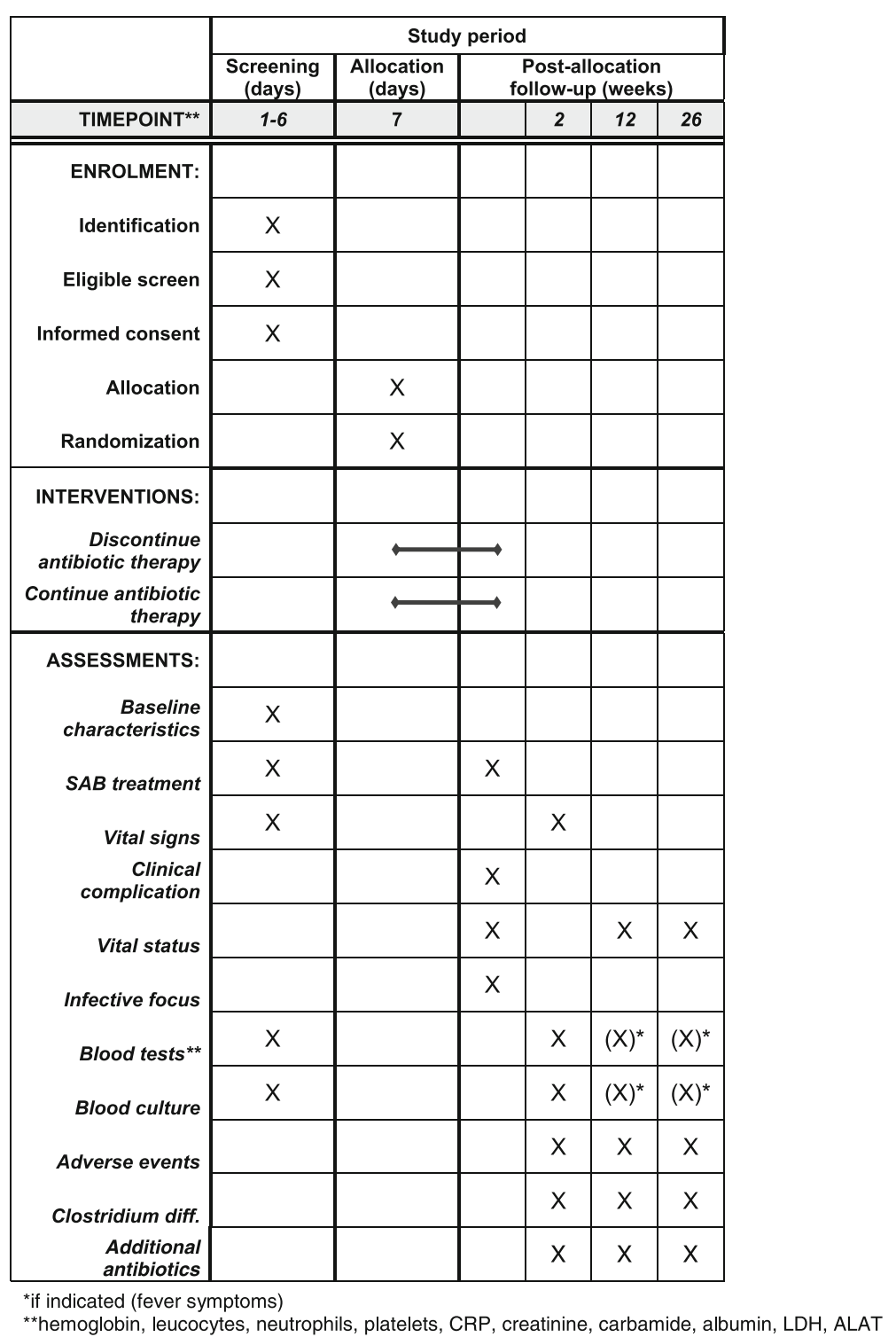

Fig. 3 Data collection and follow-up for patients enrolled in SAB7

specimen obtained only from a patient with a clinical infection. As such, routine screening of asymptomatic individuals to identify potential colonization with a resistant microorganism will not be performed.

To exclude potential effects of concomitant medical therapy not related to SAB treatment, additional antibiotic consumption within the study period is registered.

Public health-related costs will be estimated from a general consideration of the expenses associated with hospitalization for SAB.

\section{Statistics}

\section{Pilot phase}

Based on local and national databases, we expect a failure to treat and relapse rate of $<5 \%$ for patients with uncomplicated SAB alive at day 7 and a 90-day all-cause mortality rate of approximately $7 \%$, corresponding to a recovery rate of $88 \%$ [20]. However, available data on recurrence and death in this select group of patients is sparse. Consequently, an adaptive trial design is used in which the event rate is reassessed and the sample size and the non-inferiority margin adjusted if appropriate. The reassessment will occur at the second interim analysis when 100 patients have entered the study and completed 90 days of follow-up. Reassessment after the second interim analysis may lead to one of four changes to the study: 1) sample size and non-inferiority margin will remain unchanged because the event rate is $12 \%$;2) sample size and non-inferiority margin will be lowered because the event rates is $<12 \%$; 3 ) sample size will be 
increased because the event rate is $>12 \%$ while the non-inferiority margin will remain unchanged; or 4) the trial will be terminated due to safety concerns.

The regulatory authorities will be informed of the result of the pilot phase and interim analysis and potential adjustments to sample size.

\section{Sample size}

Non-inferiority is defined as a difference in primary endpoint of up to $10 \%$. Assuming a treatment response of $88 \%$, statistical power of $80 \%$, a statistical significance level of $5 \%$ and a $2 \%$ loss to follow-up rate, 284 randomized patients are require in order to exclude predefined difference in the two groups. Sample size was estimated using simulation, with the assumption of a $12 \%$ failure in both groups and a non-inferiority margin of $10 \%$.

\section{Interim analysis}

We will perform two interim analyses when 30 and 100 patients have completed the study, respectively. This serves to evaluate the primary and secondary endpoints and potential adverse events by an independent data and safety monitoring board (DSMB).

The Haybittle-Peto method is applied to demonstrate overwhelming differences between the two treatment groups that necessitate premature termination of the trail. A significant $p$ value of 0.001 in the interim analyses will correspond to a $p$ value of 0.05 in the final analysis.

\section{Discussion}

SAB represents a persistent challenge in bacterial infections. Research in this field has until now mainly focused on identifying risk factors associated with secondary complications, handling the increasing frequency of methicillin resistant Staphylococcus aureus (MRSA) infections, and, to a lesser extent, improving the present treatment strategies. The SABATO trail is currently investigating if early oral switch therapy is safe and effective for patients with low-risk SAB [21]. Even though this study may address issues with long-course intravenous treatment, results will not affect the overall consumption of antibiotics nor treatment length. To the best of our knowledge, this is the first randomized clinical study to assess reduced treatment length in SAB and the efficacy of 7 days of treatment. We chose SAB-associated death, microbiological and clinical failure to treat, and recurrence as the primary endpoint, as we feel this is the most accurate measure of the effectiveness of the treatment.

SAB7 will, regardless of the outcome, provide important information on optimized treatment of patients with uncomplicated SAB. At best, the study will provide evidence that a reduction in treatment length in uncomplicated SAB is sufficient and safe. If so, this may lead to a paradigm shift in the treatment of patients with uncomplicated SAB. Results of SAB7 will potentially improve future treatment in several ways. Reducing treatment length in uncomplicated $\mathrm{SAB}$ could induce earlier discharge from the hospital, decreased risk of hospital-acquired infections, decreased risk of severe adverse events, and an increased quality of life. Additionally, from a national and international perspective, decreasing the consumption of antibiotics will play an important role in preventing resistance development and secure future antimicrobial treatment.

\section{Trial status}

The first patient was recruited on July 20, 2018. Recruitment is expected to be completed in May 2021.

\section{Additional file}

Additional file 1: SPIRIT 2013 checklist. (DOC $122 \mathrm{~kb}$ )

\section{Abbreviations}

ALAT: Alanine amino transferase; CRP: C-reactive protein; DSMB: Data and Safety Monitoring Board; GCP: Good clinical practice; ICH: International Conference of Harmonization; LDH: Lactate dehydrogenase;

MRSA: Methicillin resistant Staphylococcus aureus; MSSA: Methicillin sensitive Staphylococcus aureus; SAB: Staphylococcus aureus bacteremia

Acknowledgements

Haakon Sandholt and Steen Ladelund for statistical support.

\section{Funding}

Financial cost is held by public funds form Danish Regions and The Ministry of Health. Funders have no role in the design of the study, data collection, data analysis, or preparation of the final manuscript.

Availability of data and materials

Not applicable.

\section{Authors' contributions}

The research question of the study was conceived by TB. TB obtained funding for the study and participated in the design of the study and the preparation of the protocol. LU drafted the protocol, coordinated the study, and participated in the study design. $J$, NF, CA, and IK participated in the design of the study. All authors have read and approved the protocol in its present form.

Ethics approval and consent to participate

We require both verbal and written consent from all eligible patients prior to inclusion in the study. The study has been approved by the Research Ethics Committee of the Capital Region of Denmark, Kongens Vaenge 2, Hilleroed, 3400 Denmark (H-17027414).

\section{Consent for publication}

Not applicable.

\section{Competing interests}

The authors declare that they have no competing interests.

\section{Publisher's Note}

Springer Nature remains neutral with regard to jurisdictional claims in published maps and institutional affiliations. 


\section{Author details}

'Department of Infectious Diseases, Hvidovre Hospital, Copenhagen University Hospital, Kettegaard Alle 30, 2650 Hvidovre, Denmark. 2Department of Clinical Microbiology, Hvidovre Hospital, Copenhagen University Hospital, Hvidovre, Denmark. ${ }^{3}$ Department of Clinical Microbiology, Rigshospitalet, Copenhagen University Hospital, Copenhagen, Denmark. ${ }^{4}$ Department of Infectious Diseases, Rigshospitalet, Copenhagen University Hospital, Copenhagen, Denmark.

Received: 27 August 2018 Accepted: 4 April 2019

Published online: 02 May 2019

\section{References}

1. Goossens H, Ferech M, Vander Stichele R, Elseviers M. Outpatient antibiotic use in Europe and association with resistance: a cross-national database study. Lancet. 2005;365(9459):579-87.

2. Lessa FC, Mu Y, Bamberg WM, Beldavs ZG, Dumyati GK, Dunn JR, et al. Burden of Clostridium difficile infection in the United States. N Engl J Med. 2015;372(9):825-34.

3. Thorlacius-Ussing L, Sandholdt H, Larsen AR, Petersen A, Benfield T. AgeDependent Increase in Incidence of Staphylococcus aureus Bacteremia, Denmark, 2008-2015. Emerg Infect Dis. maj 2019;25(5).

4. Mejer N, Westh $H$, Schønheyder HC, Jensen AG, Larsen AR, Skov R, et al. Stable incidence and continued improvement in short term mortality of Staphylococcus aureus bacteraemia between 1995 and 2008. BMC Infect Dis. 2012:17(12):260.

5. Benfield T, Espersen F, Frimodt-Møller N, Jensen AG, Larsen AR, Pallesen LV, et al. Increasing incidence but decreasing in-hospital mortality of adult Staphylococcus aureus bacteraemia between 1981 and 2000. Clin Microbiol Infect. 2007;13(3):257-63.

6. Fowler VG, Sanders LL, Sexton DJ, Kong L, Marr KA, Gopal AK, et al. Outcome of Staphylococcus aureus bacteremia according to compliance with recommendations of infectious diseases specialists: experience with 244 patients. Clin Infect Dis Off Publ Infect Dis Soc Am. 1998;27(3):478-86.

7. Blyth CC, Darragh H, Whelan A, O'Shea JP, Beaman MH, McCarthy JS. Evaluation of clinical guidelines for the management of Staphylococcus aureus bacteraemia. Intern Med J. 2002:32(5-6):224-32.

8. Nissen JL, Skov R, Knudsen JD, Ostergaard C, Schonheyder HC, FrimodtMoller $\mathrm{N}$, et al. Effectiveness of penicillin, dicloxacillin and cefuroxime for penicillin-susceptible Staphylococcus aureus bacteraemia: a retrospective, propensity-score-adjusted case?control and cohort analysis. J Antimicrob Chemother. 2013;68(8):1894-900.

9. Rasmussen JB, Knudsen JD, Arpi M, Schonheyder HC, Benfield T, Ostergaard C. Relative efficacy of cefuroxime versus dicloxacillin as definitive antimicrobial therapy in methicillin-susceptible Staphylococcus aureus bacteraemia: a propensity-score adjusted retrospective cohort study. J Antimicrob Chemother. 2014;69(2):506-14.

10. Thwaites GE, Edgeworth JD, Gkrania-Klotsas E, Kirby A, Tilley R, Török ME, et al. Clinical management of Staphylococcus aureus bacteraemia. Lancet Infect Dis. 2011;11(3):208-22.

11. Rahal JJ, Chan YK, Johnson G. Relationship of staphylococcal tolerance, teichoic acid antibody, and serum bactericidal activity to therapeutic outcome in Staphylococcus aureus bacteremia. Am J Med. 1986;81(1):43-52.

12. Mylotte JM, McDermott C. Staphylococcus aureus bacteremia caused by infected intravenous catheters. Am J Infect Control. 1987;15(1):1-6.

13. Mylotte JM, McDermott C, Spooner JA. Prospective study of 114 consecutive episodes of Staphylococcus aureus bacteremia. Rev Infect Dis. 1987;9(5):891-907.

14. Ehni WF, Reller LB. Short-course therapy for catheter-associated Staphylococcus aureus bacteremia. Arch Intern Med. 1989;149(3):533-6.

15. Raad II, Sabbagh MF. Optimal duration of therapy for catheter-related Staphylococcus aureus bacteremia: a study of 55 cases and review. Clin Infect Dis Off Publ Infect Dis Soc Am. 1992;14(1):75-82.

16. Zeylemaker MM, Jaspers CA, van Kraaij MG, Visser MR, Hoepelman IM. Longterm infectious complications and their relation to treatment duration in catheter-related Staphylococcus aureus bacteremia. Eur I Clin Microbiol Infect Dis. 2001;20(6):380-4.

17. Liu C, Bayer A, Cosgrove SE, Daum RS, Fridkin SK, Gorwitz RJ, et al. Clinical practice guidelines by the Infectious Diseases Society of America for the treatment of methicillin-resistant Staphylococcus aureus infections in adults and children. Clin Infect Dis. 2011;52(3):e18-55.
18. Shopsin B, Gomez M, Montgomery SO, Smith DH, Waddington M, Dodge $D E$, et al. Evaluation of protein A gene polymorphic region DNA sequencing for typing of Staphylococcus aureus strains. J Clin Microbiol. 1999;37(11): $3556-63$.

19. Larsen AR, Stegger M, Sørum M. spa typing directly from a mecA, spa and pvl multiplex PCR assay-a cost-effective improvement for methicillinresistant Staphylococcus aureus surveillance. Clin Microbiol Infect. 2008; 14(6):611-4.

20. Stammler Jaliff B, Dahl-Knudsen J, Petersen A, Skov R, Benfield T. Outcome and reinfection after Staphylococcus aureus bacteraemia in individuals with and without HIV-1 infection: a case-control study. BMJ Open. 2014;4(4): e004075.

21. Kaasch AJ, Fätkenheuer G, Prinz-Langenohl R, Paulus U, Hellmich M, Weiß V, et al. Early oral switch therapy in low-risk Staphylococcus aureus bloodstream infection (SABATO): study protocol for a randomized controlled trial. Trials. 2015;16:450

\section{Ready to submit your research? Choose BMC and benefit from:}

- fast, convenient online submission

- thorough peer review by experienced researchers in your field

- rapid publication on acceptance

- support for research data, including large and complex data types

- gold Open Access which fosters wider collaboration and increased citations

- maximum visibility for your research: over $100 \mathrm{M}$ website views per year

At BMC, research is always in progress.

Learn more biomedcentral.com/submissions 